\title{
WITHIN-HOST POPULATION DYNAMICS AND THE EVOLUTION OF MICROPARASITES IN A HETEROGENEOUS HOST POPULATION
}

\author{
Vitaly V. Ganusov, ${ }^{1,2}$ Carl T. Bergstrom, ${ }^{3,4}$ and Rustom Antia ${ }^{1,5}$ \\ ${ }^{1}$ Department of Biology, Emory University, Atlanta, Georgia 30322 \\ ${ }^{2}$ E-mail:vganuso@emory.edu \\ ${ }^{3}$ Department of Zoology, University of Washington, Seattle, Washington 98195 \\ ${ }^{4}$ E-mail: cbergst@u.washington.edu \\ ${ }^{5}$ E-mail: rantia@emory.edu
}

\begin{abstract}
Why do parasites harm their hosts? The general understanding is that if the transmission rate and virulence of a parasite are linked, then the parasite must harm its host to maximize its transmission. The exact nature of such trade-offs remains largely unclear, but for vertebrate hosts it probably involves interactions between a microparasite and the host immune system. Previous results have suggested that in a homogeneous host population in the absence of super- or coinfection, within-host dynamics lead to selection of the parasite with an intermediate growth rate that is just being controlled by the immune system before it kills the host (Antia et al. 1994). In this paper, we examine how this result changes when heterogeneity is introduced to the host population. We incorporate the simplest form of heterogeneity-random heterogeneity in the parameters describing the size of the initial parasite inoculum, the immune response of the host, and the lethal density at which the parasite kills the host. We find that the general conclusion of the previous model holds: parasites evolve some intermediate growth rate. However, in contrast with the generally accepted view, we find that virulence (measured by the case mortality or the rate of parasite-induced host mortality) increases with heterogeneity. Finally, we link the within-host and between-host dynamics of parasites. We show how the parameters for epidemiological spread of the disease can be estimated from the within-host dynamics, and in doing so examine the way in which trade-offs between these epidemiological parameters arise as a consequence of the interaction of the parasite and the immune response of the host.
\end{abstract}

Key words._Evolution of microparasites, host heterogeneity, immune system, virulence, within-host models.

Received April 30, 2001. Accepted September 14, 2001.

Microparasites depend critically on their hosts to ensure both their livelihood and transmission, yet many are virulent, that is, they cause harm to their hosts. Why do microparasites do this? In some cases virulence is not selected but is simply coincidental; for example, virulence observed in polio may arise due to "short-sighted" evolution of the virus resulting in the infection of neurons even though this does not increase transmission (Levin and Bull 1994). In other cases transmission, and thus reproductive success of the parasite, may be linked to the harm that it causes to its host; for example, the transmission rate of the myxoma virus may be positively correlated with its density within the host and the associated formation of lesions (Fenner and Ratcliffe 1965). Although the empirical evidence in favor of this hypothesis remains limited, linkages or correlations of this sort (frequently referred to as trade-offs) have been proposed in a number of experimental systems (Fenner and Ratcliffe 1965; Schulman 1970; Anderson and May 1982; Bull and Molineux 1992; Ewald 1993; Ebert 1994; Ebert and Herre 1996; Frank 1996; Ebert and Mangin 1997; Mackinnon and Read 1999; Messenger et al. 1999). Unfortunately, the precise shape of such trade-offs have rarely been determined (Anderson and May 1982; Ebert and Herre 1996), and the underlying causes of trade-offs between transmission and virulence are not well understood for the majority of host-parasite systems.

Broadly speaking, theoretical models of virulence evolution have typically employed several different conceptual approaches (for a comprehensive review see Frank 1996), two of which we would like to emphasize in particular. The first approach is essentially epidemiological (Anderson and May 1991). In this approach parasites evolve to maximize the net reproductive rate $R_{0}$, the average number of new infections arising from a single infected host introduced into a wholly susceptible population (May and Anderson 1983):

$$
R_{0}=\frac{\beta N}{\alpha+b+v}
$$

where $\beta$ is the transmissibility; $\alpha, b$, and $v$ are the rate constants for the parasite-induced and natural host mortality and recovery, respectively; and $N$ is the density of susceptible hosts. In the absence of superinfection, selection in the parasite population will act to maximize $R_{0}$ (May and Anderson 1983; Bremermann and Thieme 1989). If $\alpha, \beta$, and $v$ are not associated, this will lead to selection for highly transmissible $(\beta \rightarrow \infty)$, avirulent $(\alpha \rightarrow 0)$ parasites. Virulent parasites can evolve if there are trade-offs between $\alpha$ and $\beta$ or $v$, with evolved virulence levels determined by the shape of the tradeoff (Anderson and May 1982; May and Anderson 1983). Numerous extensions of the basic susceptible-infectious-recovered (SIR; Bailey 1975) approach have explored the way in which virulence evolution is affected by factors such as mutation (Bonhoeffer and Nowak 1994; Bergstrom et al. 1999), co- or superinfection (Levin and Pimentel 1981; Frank 1992; Nowak and May 1994; May and Nowak 1995; Mosquera and Adler 1998), the mode of transmission (Lipsitch et al. 1995a,b; Bergstrom et al. 1999), host susceptibility Antonovics and Thrall 1994; Frank 1994; van Baalen 1998; Gandon and Michalakis 2000), and host heterogeneity (Regoes et al. 2000).

The second approach focuses on the within-host dynamics of the parasite. These models typically track the parasite population within individual hosts and determine how factors 
that influence this within-host dynamics of the parasite will affect both virulence of the parasite and its transmission. The immunological defenses of vertebrates have evolved to combat parasites and may be expected to be one of the most important factors in determining the within-host dynamics of the parasites. Several models have explicitly considered how the interaction between the parasite and the immune response affects the within-host dynamics and the consequences for the evolution of virulence of parasites (Antia et al. 1994; Bonhoeffer and Nowak 1994a; Antia and Lipsitch 1997).

The epidemiological and within-host approaches each have their advantages and shortcomings. The major advantage of the epidemiological approach lies in its generality. By painting nature with a broad brush, these models are typically not restricted to single particular infections. This approach has been used in a number of ways. First, this approach can help determine the nature of the trade-offs between $\alpha, \beta$ and $v$ from the epidemiology of spread of specific infections such as the myxoma virus (Anderson and May 1982). Second, one can assume the existence of various trade-offs between virulence and transmissibility, and then explore the populationlevel consequences of these trade-offs (Anderson and May 1982; May and Anderson 1983). Herein lies a major shortcoming of these models, however: because they assume a priori that trade-offs exist, they cannot be used to investigate the source and nature of these trade-offs, nor to derive the existence of these trade-offs from basic biological principles. Third, the epidemiological approach can be used to explore how factors such as intrahost competition between parasite strains alter the evolved level of parasite virulence (Levin and Pimentel 1981; Bonhoeffer and Nowak 1994b; Nowak and May 1994; May and Nowak 1995; van Baalen and Sabelis 1995; Frank 1996; Mosquera and Adler 1998; Gandon et al. 2001).

Within-host models typically make much stronger assumptions about the details of infection and host response, and as such are necessarily more narrow in applicability. However, in contrast to many epidemiological models, the trade-offs between transmissibility and virulence naturally emerge from the within-host dynamics of the parasite and immune system (Antia et al. 1994). As we show in this paper, these emergent trade-offs can then, in turn, be used to understand the epidemiological properties of the parasite.

\section{Introducing Heterogeneity}

The evolution of virulence is, at its core, a coevolutionary process between parasite and host. As such, we might expect that a proper understanding of this process will require consideration of heterogeneity in both the parasite and host populations. Most previous studies have focused on how heterogeneous parasites will evolve in a population of homogeneous hosts (e.g., Nowak and May 1994). More complex extensions of these models also incorporate host heterogeneity with differences in host susceptibility, host recovery rates, and the ability to transmit the parasite. (These and other forms of host heterogeneity have been observed in a number of host-parasite systems; e.g., Fenner and Ratcliffe 1965; Anderson and May 1982; May and Anderson 1983; Ebert and Hamilton 1996; Zhong and Dobson 1996; Woolhouse et al. 1997). However, these models have largely been aimed at explaining the observed stable polymorphism in host susceptibility and parasite virulence found for many parasitehost systems (Antonovics and Thrall 1994; Frank 1994; Gupta and Hill 1995; Morand et al. 1996). The question of how host heterogeneity affects the evolution of parasite virulence has, until recently, been less thoroughly explored. In a comprehensive review, Ebert and Hamilton (1996) suggested that parasites evolving in heterogeneous populations should evolve to be less virulent than parasites adapting to one host type; a parasite that is specialized to exploit one host type will do poorly at exploiting other hosts. Therefore, if more than one different host population is infected by a particular parasite strain, virulence cannot increase in all of the infected populations simultaneously. Regoes et al. (2000) derived a similar conclusion from a theoretical model of parasite evolution in heterogeneous populations, and further explored how, depending on the trade-off between virulence of the parasite in two host types, parasites may evolve to be generalists, infecting hosts of both types equally well, or specialists, infecting one host type to the exclusion of the other.

The addition of heterogeneity to the within-host models of acute infections is particularly important because the simple model predicts that to maximize transmission, the growth rate of the parasite will evolve to be sufficiently high that maximum parasite density falls just short of the lethal density (the density of the parasite at which it kills the host) before it is cleared by the immune response (Antia et al. 1994). In this case, the evolved parasite does not kill its host. Because the optimum is at this knife edge, the introduction of even a little random or stochastic heterogeneity in the parameters could substantially change the level of parasite virulence. Indeed, Antia and Lipsitch (1997) show that stochastic heterogeneity in the host population results in a decrease in both the optimal growth rate of the parasite and the total transmission. However, they did not consider the consequences of host heterogeneity on the evolution of parasite virulence.

In this paper we introduce a simple type of heterogeneity, random heterogeneity in the parameters describing the host response, and explore how it affects parasite evolution. We expect random heterogeneity to be a virtually ubiquitous aspect of the host-parasite interactions. For example, there may be stochastic variation in the initial parasite inoculum, and hosts may, as a result of being exposed to different antigenic and nutritional environments, have small differences in their specific immune responses following infection (Traub 1936; Fenner and Ratcliffe 1965; Schulman 1970; Zhong and Dobson 1996; Woolhouse et al. 1997). We do not consider the sort of large-scale host heterogeneity that results in specialist/ generalist trade-offs such that parasite adaptations favored in one host type necessarily engender fitness costs in the other host type(s). We show that, as expected, the introduction of heterogeneity in the host population prevents parasite adaptation to a particular host type and this results in a decrease in the average transmission of the parasite from an infected host. We find, however, that while an increase in heterogeneity initially leads to a decrease in the optimal growth rate of the parasite, this decline is not monotonic - at very high levels of heterogeneity the optimal growth rate begins to increase. The most interesting and surprising results concern 
A

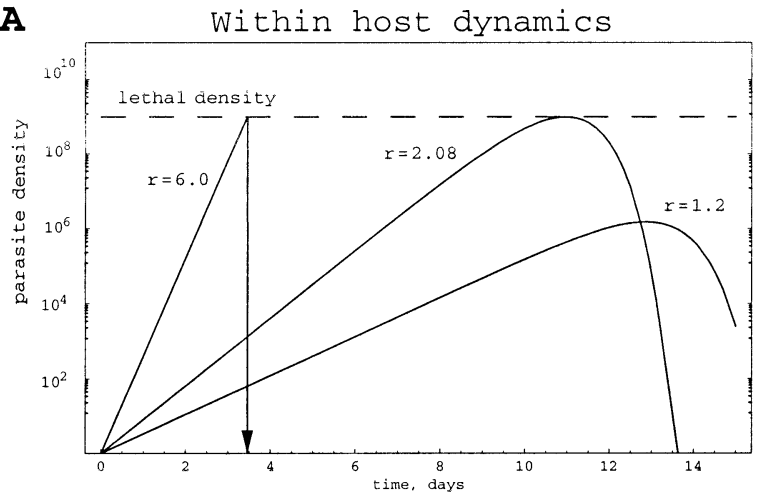

B

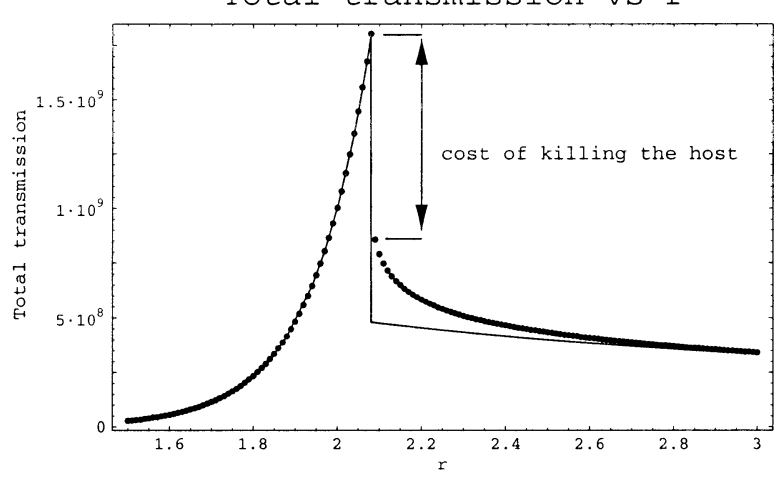

FIG. 1. The evolution of microparasites in the absence of host heterogeneity. (A) The within-host dynamics of parasites with different growth rates, $r$. Slowly growing parasites $(r=1.20)$ are cleared by the immune system before they reach a high density, rapidly growing parasites $(r=6.0)$ kill the host before the immune response is activated, and parasites with intermediate growth rates $(r=2.08)$ just approach lethal density, $D$, before clearance by the immune system. (B) The total transmission of a parasite over the duration of infection as a function of its growth rate, $r$. The maximum total transmission corresponds to the growth rate at which the parasite is just controlled by the immune system before it reaches $D$. A numerical solution of equation $(2)(\cdots)$ and analytical approximation given by equation (5) $(-)$ are shown. $P_{0}=1, X_{0}=1, h=10^{-3}, k=10^{3}, D=10^{9}, s=1$.

the evolution of virulence in heterogeneous populations. We find that contrary to conventional expectations (Ebert and Hamilton 1996; Ebert 1998; Regoes et al. 2000), in our model changes in virulence depend critically on how virulence is measured; virulence measured by the case mortality increases rather than decreases as heterogeneity is increased. Finally we show how the parameters for epidemiological spread of the disease can be estimated from the within-host dynamics of parasites, and in doing so we examine the trade-offs between the epidemiological parameters $\alpha, \beta$, and $v$ that result from the within-host model.

\section{RESUlts AND Discussion}

\section{Formulation of the Mathematical Model}

We employ a model introduced by Antia et al. (1994) that is designed to describe acute infections in vertebrate hosts with rapidly growing microparasites. In this model, the cycle of infection, transmission, and clearance proceeds as follows: (1) infection is initiated in a new host by a fixed inoculum, $P_{0}$, and the parasite population grows exponentially in the absence of a specific immune response; (2) the presence of the parasite induces a specific immune response in the host, which grows by clonal expansion in a parasite-dependent manner and kills the parasite at a rate proportional to the product of the parasite and immune cells densities; (3) there is a lethal, or threshold, density of parasite, $D$, at which infection kills the host; and (4) the rate of transmission of the parasite from an infected host is proportional to the parasite density within the host, and we assume that the parasite is selected to maximize its total transmission from an infected host during the course of the acute infection. Given these assumptions, the rates of change in the densities of the parasite $(P)$ and specific immune cells $(X)$ will be given by the following equations:

$$
\begin{aligned}
& \frac{d P}{d t}=r P-h P X \quad \text { and } \\
& \frac{d X}{d t}=\frac{s X P}{k+P},
\end{aligned}
$$

where $r$ and $s$ are the maximum growth rates of the parasite and immune cells, respectively; $h$ is the rate constant for clearance of the parasite by the immune response; and $k$ is the parasite density that stimulates immune cells to grow at half their maximum rate. Because we are primarily concerned with the dynamics of parasites during acute infections, we ignore the contraction and memory phases of the immune response that occur following control and clearance of the parasite. Biologically the relative magnitudes of various parameters are (Antia et al. 1994):

$$
\begin{aligned}
P_{0} & \ll k \ll D \text { and } \\
h X_{0} & \ll r, s,
\end{aligned}
$$

where $X_{0}=X(0)$ is the precursor frequency (the initial number of immune cells specific to the parasite). The within-host dynamics of the parasites with different growth rates are illustrated in Figure 1A. We see that slowly growing parasites are cleared by the immune response before they reach a high density; parasites with intermediate growth rates reach higher densities but are cleared by the immune response, provided the lethal density, $D$, is not exceeded; and parasites with the highest growth rates reach the lethal density rapidly and kill the host before being controlled by the immune response.

If the rate of parasite transmission is proportional to the within-host density of the parasite, then a plot of the total transmission as a function of the growth rate, $r$, of the parasite shows that maximum total transmission occurs at an intermediate growth rate $r^{*}$ (Fig. 1B). The total transmission increases with increasing growth rate, provided that the lethal density is not exceeded (i.e., when $r \leq r^{*}$ ). At the point where the lethal density is just exceeded, transmission suddenly drops, because there is no transmission from the dead host (we call this drop in transmission "the cost of killing the host'). A further increase in the growth rate leads to a gradual decline in the total transmission. We can estimate the optimal growth rate, $r^{*}$ (at which the total transmission is maximum), and how the total transmission, $l(r)$, changes with the growth rate by the following expressions, derived in the Appendix: 


$$
\begin{aligned}
& \left(\frac{D}{k}\right)^{s} \approx\left(\frac{r^{*}}{h e X_{0}}\right)^{r^{*}}, \quad \text { and } \\
& l(r)=c \int_{0}^{\infty} P(t) d t \approx c \cdot \begin{cases}\frac{k}{s}\left(\frac{s}{h X_{0}}\right)^{r / s} \Gamma\left(\frac{r}{s}\right), & \text { if } r \leq r^{*} \\
D / r, & \text { if } r>r^{*},\end{cases}
\end{aligned}
$$

where $e$ is the base of the natural logarithm.

What consequences does this have for the evolution of virulence? The answer depends on how virulence is defined. We consider two commonly used measures for virulence, the $L D_{50}$ and the case mortality. (The $L D_{50}$, or lethal dose 50 , is the initial dose of the parasite required to kill $50 \%$ of infected hosts in a fixed period of time [Davis et al. 1969]. The case mortality is the probability of host death due to the infection.) If we use the $L D_{50}$ as the measure of virulence, we find that the model predicts that the $L D_{50}$ varies inversely with the growth rate, $r$, and selection for an intermediate growth rate implies selection for intermediate levels of virulence (see Antia et al. 1994). If we instead measure virulence by the case mortality, the model predicts that virulence of a fully evolved parasite (with $r=r^{*}$ ) is zero because the maximum parasite density will always fall just short of the lethal density. Because maximum transmission occurs when the maximum parasite density falls infinitesimally short of the lethal density, the level of virulence may change dramatically if any heterogeneity is introduced. How it will change is addressed in the next section.

\section{Evolution in a Heterogeneous Environment}

In the simplest model we assumed that, except for the parasite growth rate, $r$, all the parameters describing the parasite-host interaction are fixed. In this section we introduce random heterogeneity of the parameters describing the host response, and ask how the optimal growth rate, total transmission, and virulence level of the parasite change with the amount of heterogeneity present in the host population. Heterogeneity in host response to parasite pressure may take a number of (not mutually exclusive) forms. For example, hosts may vary in immune-cell activation rates (different $k$ ), frequencies of precursor immune cells (different $X_{0}$ ), immunecell specificity to the parasite $(h)$, and threshold densities at which parasite load becomes lethal $(D)$. For simplicity, we will begin the analysis by introducing heterogeneity in only one parameter, the threshold density, $D$, at which the parasite kills the host. We do so by generating a distribution of threshold densities for a particular host population described by the probability density function $f(D)$ such that $f(D) d D$ is the probability that a given host has lethal density in a range $(D$, $D+d D)$. We consider two simple distributions of the probability density function $f$ : uniform and gamma distributions. We characterize the degree of host heterogeneity by the coefficient of variation, $C V=\sqrt{\sigma^{2}} /\langle D\rangle$, where $\langle D\rangle$ is the mean and $\sigma^{2}$ is the variance of the threshold density. As in the simple model, we assume that different parasite strains differ only in their growth rate, $r$, and that $r$ evolves so as to maximize the total transmission over the course of an infection.
The average (expected) total transmission of a parasite with growth rate $r$ in this heterogeneous host population is given by the integral of the product of the probability density function, $f(D)$, and the instantaneous transmission rate, $l(r, D)$ over the course of the infection:

$$
L(r)=\int_{0}^{\infty} l(r, D) f(D) d D .
$$

Substituting $l(r, D)$ from equation (5) we find:

$$
\begin{aligned}
L(r) \approx & \frac{k c}{s}\left(\frac{s}{h X_{0}}\right)^{r / s} \Gamma\left(\frac{r}{s}\right) \int_{k\left(r /\left(h e X_{0}\right)\right)^{r / s}}^{\infty} f(D) d D \\
& +\frac{c}{r} \int_{0}^{k\left(r / h e X_{0}\right)^{r / s}} D f(D) d D .
\end{aligned}
$$

(This approximation has been used to generate the curves in Figures 2 and 4.)

In the subsequent sections, we explore in detail the consequences of heterogeneity in the parameter $D$. The discussion of how heterogeneity in other parameters affects the evolution of microparasites is relegated to the end of this section.

\section{Transmission and the optimal growth rate}

The first two panels of Figure 2 provide an overview of the consequences of introducing heterogeneity in the lethal density, $D$. The total transmission changes as a function of growth rate for different levels of uniformly distributed (Fig. $2 \mathrm{~A}$ ) or gamma distributed (Fig. 2B) heterogeneity. Both the total transmission and the growth rate at which transmission is maximized change with increasing heterogeneity. For both uniform and gamma distributions: the total transmission decreases monotonically with increasing heterogeneity, and the optimal growth rate, $r_{o p t}$, appears to first decrease and then increase as heterogeneity increases. These results are shown in more detail in Figures $2 \mathrm{C}$ and 2D. The decrease in the total transmission with increasing heterogeneity is, in retrospect, unsurprising. As heterogeneity increases, the parasite is not able to optimize as well as when there is a single host type. The change in the parasite's optimal growth rate from the value $r^{*}$, which the parasite has in the absence of heterogeneity, to $r_{o p t}$, which it has in the presence of heterogeneity, is somewhat less intuitive. This change is a consequence of the asymmetry of the total transmission around the optimum $r^{*}$ observed in Figure 1B. At small departures from the growth rate $r^{*}$, higher transmission is obtained by slightly undercutting the growth rate $r^{*}\left(r_{o p t}<r^{*}\right)$ than by exceeding it. At higher departures from the optimum, higher transmission can obtained by exceeding the growth rate $r^{*}$ than by undercutting it. Thus, when heterogeneity is low, it is on average better to have a slightly lower than the growth rate $r^{*}$; when heterogeneity is high, it is better to have a higher growth rate.

\section{Evolution of virulence}

We now examine the effects of host heterogeneity on virulence evolution. We measure virulence in two different ways, as the case mortality, $M$, and the $L D_{50}$. Figure $2 \mathrm{E}$ shows 
Transmission vs growth rate
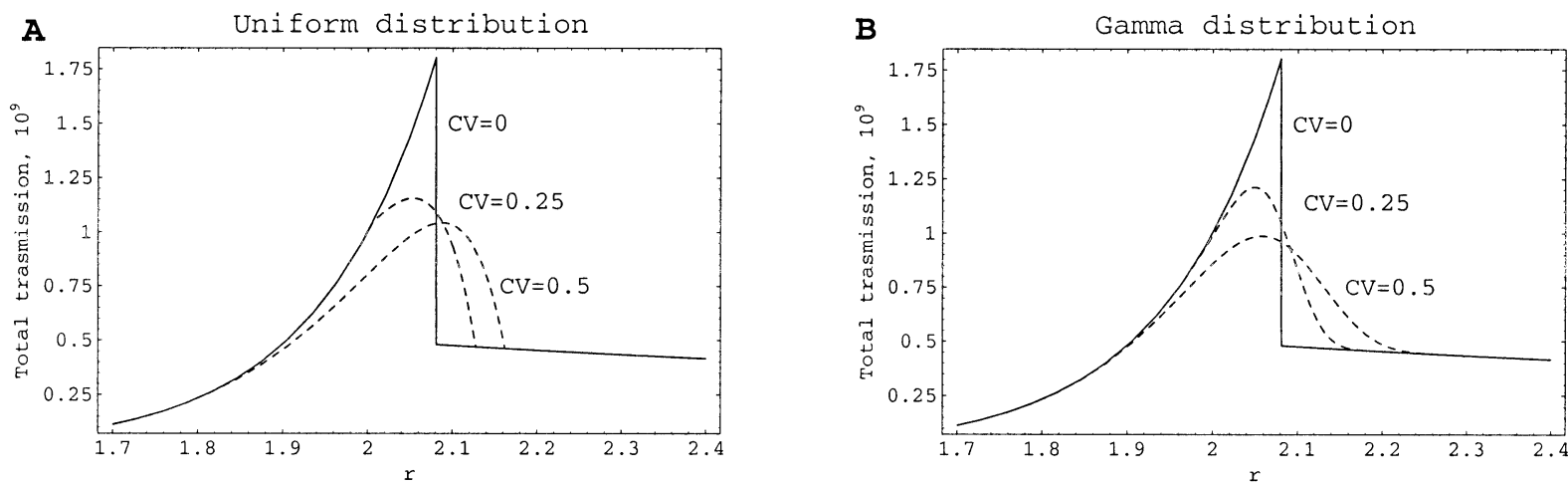

Transmission and growth rate vs heterogeneity
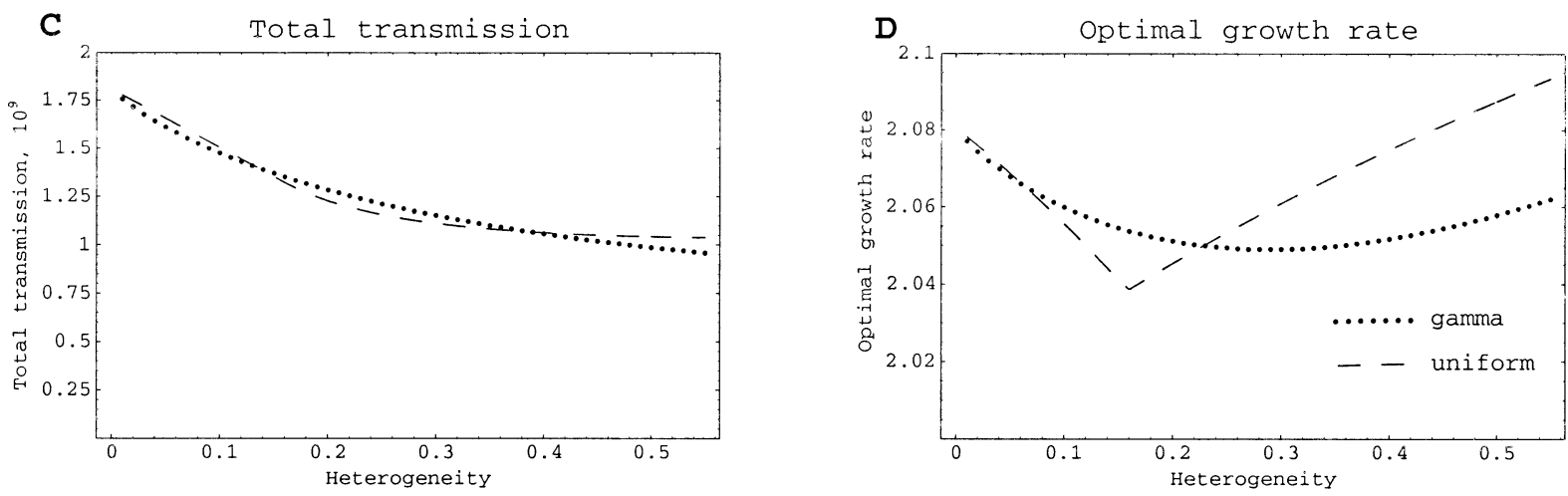

Virulence vs heterogeneity
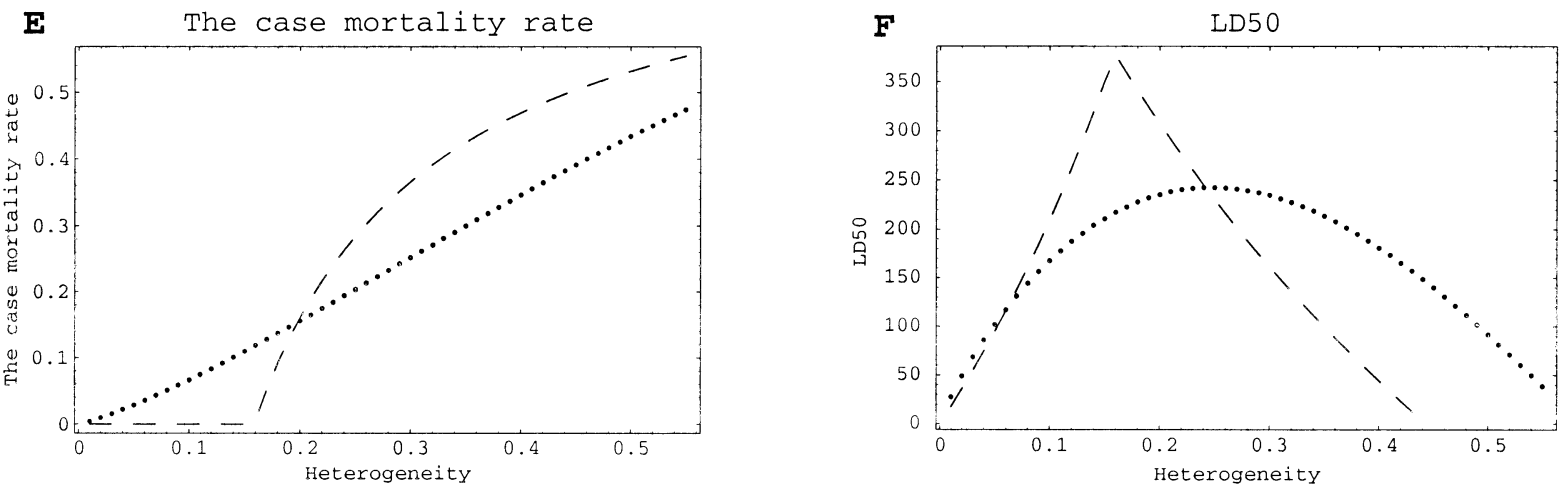

FIG. 2. The evolution of microparasites in a heterogeneous host population. We examine the effects of adding heterogeneity in the lethal density, $D$, on the evolution of parasites. (A, B) The changes in the total transmission of parasites with different growth rates, $r$, when host heterogeneity in the lethal density is modeled by uniform or gamma distributions, respectively, in the absence ( - ) and presence (- - ) of heterogeneity $(C V$ is marked). (C-F) The consequences of heterogeneity in the lethal density for the total transmission (C), the optimal growth rate (D), case mortality (E), and $L D_{50}(\mathrm{~F})$. Host heterogeneity is modeled by uniform (- - or gamma ( $\cdots$ ) distributions. See Appendix for details of the calculations. Parameters are the same as in Figure 1, with $\langle D\rangle=10^{9}$.

how the case mortality changes with increasing levels of host heterogeneity. For both uniform and gamma distributions of threshold density values, the case mortality increases from zero as heterogeneity increases. When threshold density, $D$, is uniformly distributed, there is an initial period when the heterogeneity is low during which there is no increase in the case mortality. When threshold densities are given by a gamma distribution, the case mortality increases almost linearly 
with increasing heterogeneity. The increase in case mortality is determined by two factors. The optimal growth rate, $r_{\mathrm{opt}}$, decreases, reducing the maximum density of the parasite within the host. However, heterogeneity in the lethal density, $D$, results in some fraction of the host population having a sufficiently low lethal density that they are killed before the immune response controls the parasite.

In other words, the case mortality increases with increasing heterogeneity as a consequence of the following two changes. First, increasing heterogeneity in $D$ (when the parasite growth rate, $r$, is fixed and is below the optimal value, $r^{*}$, given by eq. 4) results in an increasing fraction of hosts having a peak parasitemia above the lethal density, $D$; this leads to increasing case mortality. Second, introducing heterogeneity changes the optimal growth rate $r_{o p t}\left(r_{o p t}=r^{*}\right.$ when heterogeneity is zero). All else being equal, decreases in $r_{\text {opt }}$ lead to a reduction in the peak parasitemia; this leads to decreasing case mortality. As heterogeneity increases from zero to higher values, these two changes pull the case mortality in opposite directions, and for nonlinear equations we find it difficult to intuit the net result. The results of our analysis show that the net effect is an increase in case mortality with increasing heterogeneity.

Figure $2 \mathrm{~F}$ shows how the $L D_{50}$ changes with increasing heterogeneity. In the absence of heterogeneity, the $L D_{50}$ is just above the initial parasite density, $P_{0}$. As heterogeneity increases, the $L D_{50}$ first increases and then decreases. Changes in the $L D_{50}$ arise as a consequence of the changes in the optimal growth rate of the parasite as heterogeneity increases; the $L D_{50}$ varies inversely with the parasite's growth rate, $r$ (see Appendix for details).

Taken together, Figures $2 \mathrm{E}$ and $2 \mathrm{~F}$ highlight the critical dependence of our qualitative conclusions regarding virulence evolution on the way in which we choose to measure virulence. The case mortality is the more appropriate measure of virulence because it more closely reflects the actual host mortality caused by the infection and thus the reduction in the host's fitness (i.e., virulence), whereas the $L D_{50}$ is an indirect measure of virulence. In addition, our results show clearly that virulence of a parasite measured by the case mortality does not necessarily decrease as host heterogeneity increases.

\section{Heterogeneity in other parameters}

We now briefly describe the consequences of introducing heterogeneity in the other parameters describing the interaction of the parasite with the immune response. We do so by looking at how the introduction of heterogeneity in the other parameters changes the total transmission, the optimal growth rate of the parasite, and its virulence. (In this paper we restrict our analysis to the addition of heterogeneity in one parameter at a time and do not consider addition of heterogeneity in several parameters simultaneously.)

As it can be seen in Figure 3, the addition of heterogeneity in the parameters $h, X_{0}$, and $k$ gives similar results to those arising from variation in $D$ described previously.

The addition of heterogeneity in the size of initial inoculum, $P_{0}$, leads to much smaller changes in the total transmission, the optimal growth rate of the parasite, and its vir- ulence. Although these changes are too small to be seen when plotted with changes in the other parameters, they follow the same trend (results not shown). For example, total transmission decreases monotonically with increasing heterogeneity in $P_{0}$. In contrast, the addition of heterogeneity in the growth rate, $r$, leads to larger changes (in total transmission, optimal growth rate, and virulence) in comparison with those observed following the addition of heterogeneity to $D$.

Only in the case of heterogeneity in the maximum growth rate of immune cells, $s$, we find that whereas the behavior is qualitatively similar to that for the other parameters at low levels of heterogeneity, it is qualitatively different when heterogeneity is large. The difference arises because, when heterogeneity in $s$ is large, there are some hosts with very small $s$, and in these hosts the immune response develops very slowly and allows for prolonged transmission of parasites with low rates of growth. Consequently at very high levels of heterogeneity in $s$, the $r_{\text {opt }}$ declines, the total transmission begins to increase, and the case mortality drops. We note that when $s$ is small the infection ceases to be an acute infection of short duration.

\section{Estimating Epidemiological Parameters}

In the introduction, we contrasted the within-host approach taken in the sections Formulation of the Mathematical Model and Evolution in a Heterogeneous Environment with the epidemiological approach commonly employed to study virulence evolution. In this section, we derive the connection between these two modeling approaches and demonstrate that the epidemiological parameters of parasite transmission depend explicitly on the within-host dynamics. From the withinhost dynamics, we can calculate the epidemiological parameters which define the basic reproductive rate of the parasite (eq. 1), namely the rate of parasite transmission from infected to susceptible hosts, $\beta$, and the rates of parasite-induced host mortality, $\alpha$, and recovery, $v$, and examine the resultant tradeoffs between these epidemiological parameters. The epidemiological parameters can be obtained from the within-host dynamics as follows.

First, the basic reproductive number, $R_{0}$, is proportional to the average number of parasites transmitted from an infected host over the course of acute infection, that is, $R_{0}(r)=u L(r)$, where $u$ is a coefficient of proportionality. Second, the transmission rate of a parasite with the growth rate $r$ from a host with a lethal density $D$ equals the total transmission of the parasite over the course of acute infection, $l(r, D)$, divided by the duration of acute infection, $\Delta(r, D)$ :

$$
\beta(r, D)=u \frac{l(r, D)}{\Delta(r, D)},
$$

where $l(r, D)$ is given by equation $(5)$ and $\Delta(r, D)$ is derived in the Appendix. The average transmission rate of the parasite, $\beta(r)$, from the host population with heterogeneity described by the distribution $f(D)$ is:

$$
\begin{aligned}
\beta(r) & =\int_{0}^{\infty} \beta(r, D) f(D) d D \\
& =u \int_{0}^{\infty} \frac{l(r, D)}{\Delta(r, D)} f(D) d D .
\end{aligned}
$$


Transmission and growth rate vs heterogeneity
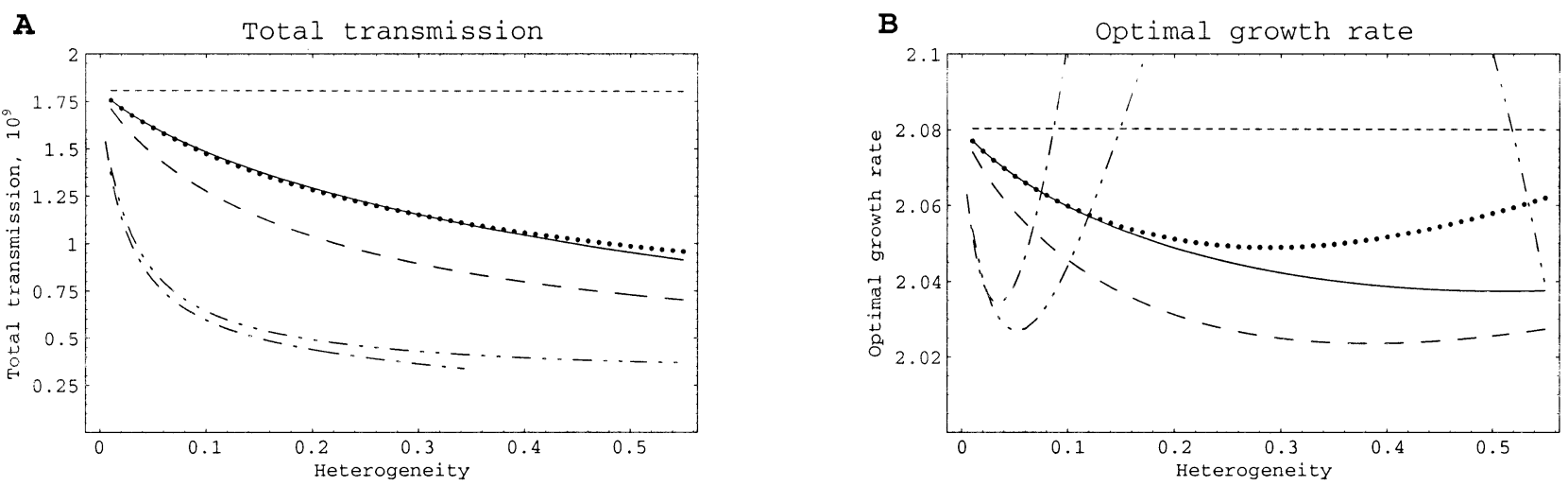

Virulence vs heterogeneity

C

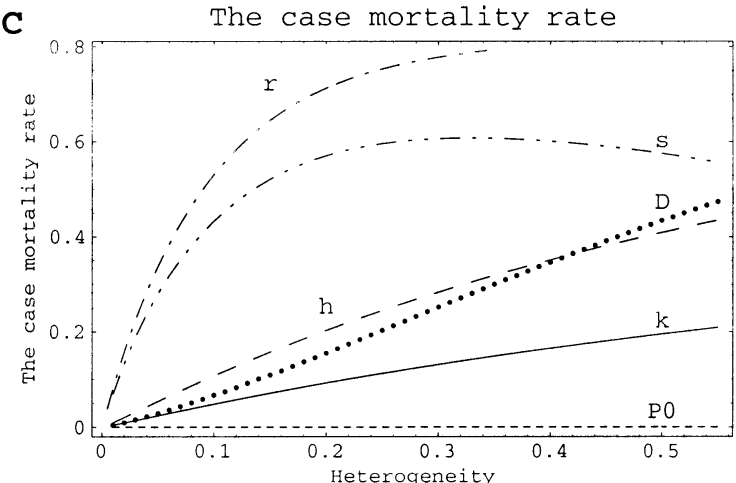

D

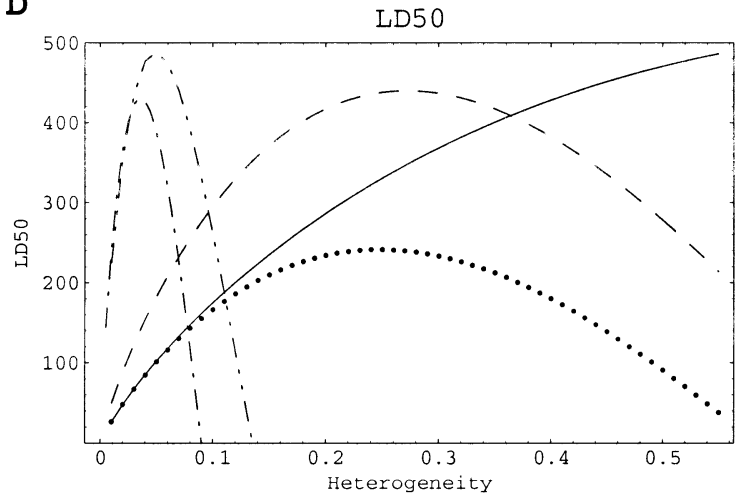

FIG. 3. Consequences of heterogeneity in other parameters on the evolution of microparasites. We compare the consequences of heterogeneity in the different parameters for the total transmission (A), the optimal growth rate (B), case mortality (C), and $L D_{50}(\mathrm{D})$. Heterogeneity in $D(\cdots), k(-), h\left(X_{0}\right)(---), P_{0}(--), r(-\cdot-)$, and $s(-\cdots-)$ is modeled by a gamma distribution. Parameters are the same as in Figure 1, with average values: $\left\langle P_{0}\right\rangle=1,\left\langle X_{0}\right\rangle=1,\langle h\rangle=10^{-3},\langle k\rangle=10^{3},\langle D\rangle=10^{9},\langle s\rangle=1$.

The mortality rate, $\alpha$, equals the rate of parasite-induced host death following infection. If hosts die following infection, then $\alpha$ is inversely proportional to the average duration of the infection. If hosts survive the infection then $\alpha$ is zero. If $m(r, D)$ equals the case mortality (derived in the Appendix) and $\Delta(r, D)$ equals the duration of infection, we get

$$
\alpha(r, D)=\frac{m(r, D)}{\Delta(r, D)} .
$$

Similarly, the recovery rate of hosts with a lethal density $D$ infected with a parasite with growth rate $r$ is

$$
v(r, D)=\frac{1-m(r, D)}{\Delta(r, D)} .
$$

The average recovery and mortality rates of the host population with heterogeneity described by the distribution $f(D)$ are found similarly to the average transmission rate $\beta(r)$ :

$$
\alpha(r)=\int_{0}^{\infty} \alpha(r, D) f(D) d D=\int_{0}^{D^{*}} \frac{f(D)}{\Delta(r, D)} d D,
$$

and

$$
v(r)=\int_{D^{*}}^{\infty} \frac{f(D)}{\Delta(r, D)} d D,
$$

where $D^{*}=k\left(r /\left(h e X_{0}\right)\right)^{r / s}$

Using the derived expressions for the epidemiological parameters we illustrate how $\beta, \alpha$, and $v$ change as a function of the growth rate of the parasite $r$ when host heterogeneity is held at a fixed level and the trade-offs between these parameters for parasites with different growth rates (Fig. 4).

Figure 4A shows the virulence and recovery rates. We see that the mortality rate, $\alpha$, increases with the parasite's growth rate, $r$; the rate of increase is greatest when $r$ is close to its optimal value at a given level of host heterogeneity $\left(r_{\text {opt }} \approx\right.$ 2.06 for $C V_{D}=0.50$ ). The average rate of recovery, $v$, initially decreases only gradually with increasing $r$, as the duration of infection gets slightly shorter. Thereafter, as more 
Virulence, recovery and transmission rates vs growth rate
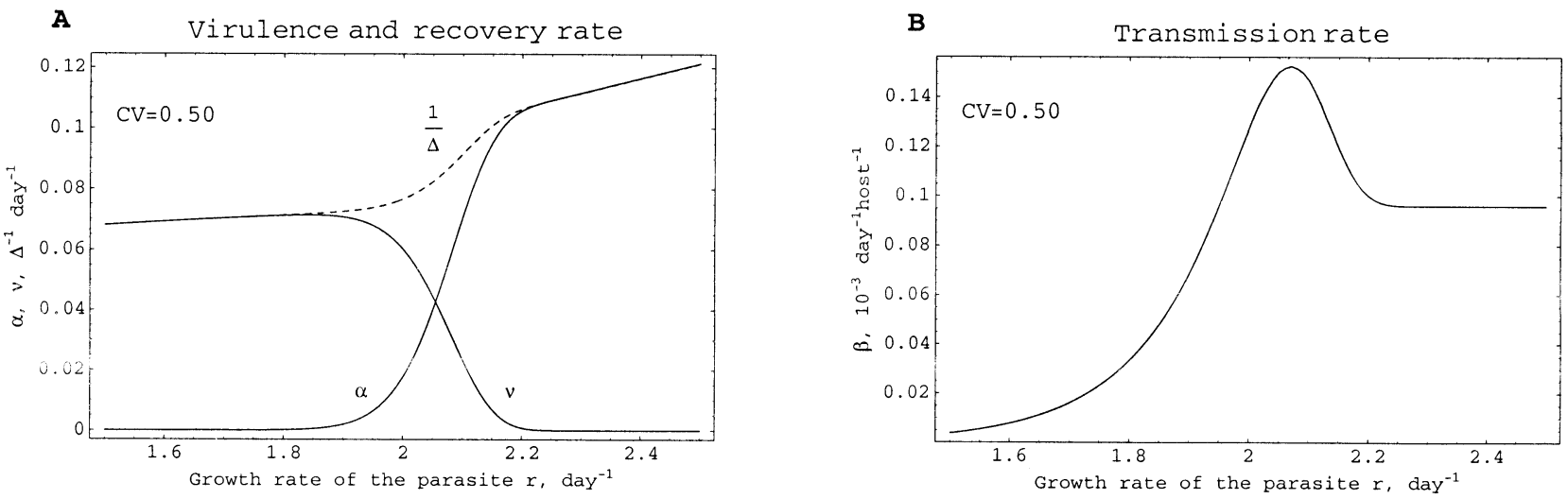

Basic reproductive number and the rate of recovery vs virulence

C

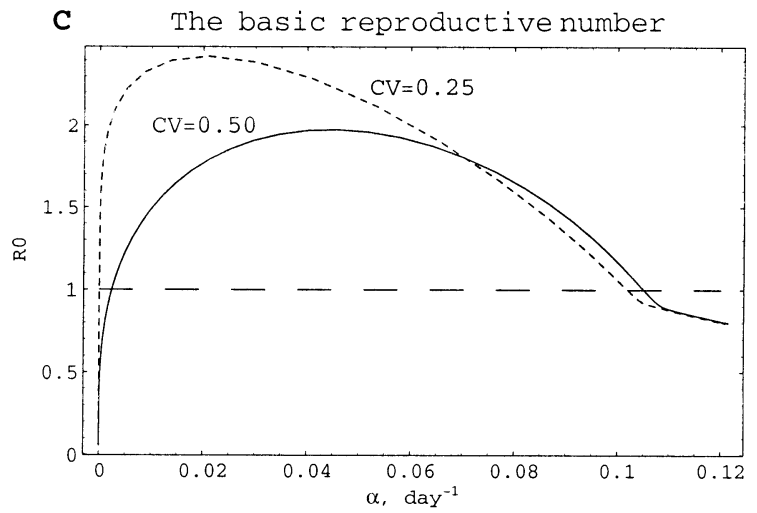

$\mathrm{D}$

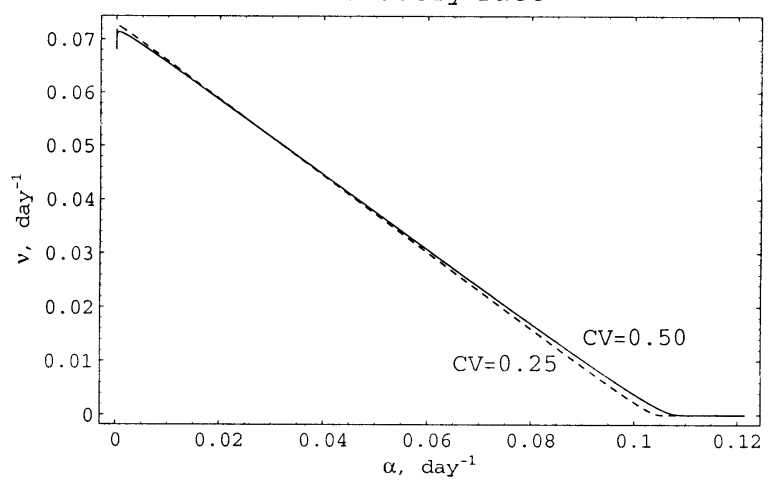

FIG. 4. Estimating epidemiological parameters from the within-host model. We estimate the epidemiological parameters for parasites with different growth rates in a heterogeneous host population $(C V=0.5)$. (A) The parasite-induced host mortality rate, $\alpha$, and the rate of host recovery, $v$. (B) The transmission rate, $\beta$. (C, D) The epidemiological trade-offs arising as a consequence of the within-host dynamics for two levels of host heterogeneity (with $C V=0.5[-]$ and $C V=0.25[---]$ ). (C) How the basic reproductive number, $R_{0}$, changes with virulence, $\alpha$. (D) The trade-off between the recovery rate, $v$, and the parasite-induced host mortality rate, $\alpha$. Parameters are the same as in Figure 2, except $N=10^{3}$ and $u=2 \times 10^{-12}$; host heterogeneity in $D$ is modeled by a gamma distribution.

hosts die, the rate of recovery rapidly declines. Figure $4 \mathrm{~B}$ shows that the transmission rate, $\beta(r)$, increases with $r$ until the growth rate at which transmission is maximum, and thereafter drops to $u\langle D\rangle / \ln \langle D\rangle$. We note that because $R_{0}(r)$ is directly proportional to the total transmission, the change in this measure as a function of growth rate corresponds to the graph for the total transmission, $L(r)$, as shown in Figures $2 \mathrm{~A}$ and $2 \mathrm{~B}$.

Finally, we visualize some of the trade-offs between the basic reproductive number, $R_{0}$, the mortality rate, $\alpha$, and the recovery rate, $v$. We focus on those trade-offs that have been explored in the epidemiological literature (Fenner and Ratcliffe 1965; Anderson and May 1982). In Figure 4C we show how the basic reproductive number, $R_{0}$, changes with the level of virulence, $\alpha$. We find that $R_{0}$ is maximized at intermediate levels of virulence. In Figure 4D we show the tradeoff between virulence and the recovery rate and find a nearly linear relationship between these parameters. The shapes of both these trade-offs are qualitatively consistent with those estimated from experimental data of the myxomatosis infec- tion in rabbits in Australia (Fenner and Ratcliffe 1965; Anderson and May 1982).

\section{Summary}

In this paper we have used a simple mathematical model to analyze how host heterogeneity affects the within-host dynamics and evolution of microparasites in vertebrate hosts. We introduce a simple form of heterogeneity, the random heterogeneity that arises inevitably in host populations due to factors such as stochastic variation in the initial density of the parasite; the precursor frequency of immune cells specific for the parasite; as well as the parameters $h, k$, and $D$, which determine the within-host dynamics of the parasite. We find that: (1) parasites evolve to an intermediate growth rate, which changes with increasing heterogeneity; (2) the total transmission of the evolved parasite decreases as heterogeneity increases; (3) the observed pattern of virulence evolution is sensitive to the measure of virulence employed (case mortality vs. $L D_{50}$, the former of which provides the 
preferable measure); (4) in contrast to the generally accepted view, virulence does not necessarily decrease with increasing heterogeneity, and indeed is likely to increase with increasing heterogeneity; and (5) the parameters for epidemiological spread of the disease can be estimated from the within-host dynamics, and in doing so we can examine the trade-offs between these epidemiological parameters that result from the interaction of the parasite and the immune response of the host.

Our finding that parasites should cause more damage to the host population when hosts are heterogeneous seems to contradict the widespread belief that the evolution of parasites in heterogeneous populations should select for less virulent parasites (Ebert and Hamilton 1996; Ebert 1998; Regoes et al. 2000). The different predictions of the earlier papers and our model arise from the way heterogeneity is introduced. In the previous papers heterogeneity in the host population implicitly imposes a trade-off on the parasite. The consequence of this trade-off is that doing well in one type of hosts impairs the fitness of the parasite when it infects the other type of hosts. In our model, we only introduce random or stochastic heterogeneity in the parameters (i.e., we do not impose any specific trade-offs).

In reality we could find both types of heterogeneity. Random heterogeneity (i.e., small differences in parameters governing the host-parasite interactions) is expected to be present in any host population due to phenotypic (or other) differences between individual hosts. When this happens, our model predicts that virulence, measured by the case mortality rate, will increase with increasing random heterogeneity. Trade-off heterogeneity may be found in other cases. The evidence, suggesting that trade-off heterogeneity exists includes serial passage experiments in which the increase in virulence of parasites when they are serially passaged through one host type is frequently linked with a decrease in the parasites virulence in another host type (Ebert 1998). Ultimately, our understanding of virulence evolution will benefit from incorporation of both random and trade-off forms of heterogeneity into evolutionary models, so as to assess the relative importance of these two sources of variation.

\section{ACKNOWLEDGMENTS}

We thank M. Tanaka and D. Genereux for their extensive comments and discussions. This work was supported by National Institutes of Health grant R29-GM-54268 to RA.

\section{Literature Cited}

Anderson, R. M., and R. M. May. 1982. Coevolution of hosts and parasites. Parasitology 85:411-426.

1991. Infectious diseases in humans: dynamics and control. Oxford Univ. Press, Oxford, U.K.

Antia, R., and M. Lipsitch. 1997. Mathematical models of parasite responses to host immune defenses. Parasitology 115: S155-S167.

Antia, R., B. R. Levin, and R. M. May. 1994. Within-host population dynamics and the evolution and maintenance of microparsite virulence. Am. Nat. 144:457-472.

Antonovics, J., and P. Thrall. 1994. The cost of resistance and maintenance of genetic-polymorphism in host-pathogen systems. Proc. R. Soc. Lond. B. 257:105-110.
Bailey, N. T. J. 1975. The mathematical theory of infectious diseases and its application. Griffin, London.

Bergstrom, C. T., P. McElhany, and L. A. Real. 1999. Transmission bottlenecks as determinants of virulence in rapidly evolving pathogens. Proc. Natl. Acad. Sci. USA 96:5095-5100.

Bonhoeffer, S., and M. Nowak. 1994a. Intra-host versus inter-host selection: viral strategies of immune function impairment. Proc. Natl. Acad. Sci. USA 91:8062-8066.

. 1994b. Mutation and the evolution of virulence. Proc. R. Soc. Lond. B. 258:133-140.

Bremermann, H. J., and H. R. Thieme. 1989. A competetive exclusion principle for pathogen virulence. J. Math. Biol. 27: 179-190.

Bull, J. J., and I. J. Molineux. 1992. Molecular genetics of adaptation in an experimenta model of cooperation. Evolution 46: 882-895.

Davis, B. D., R. Dulbecco, H. N. Eisen, H. S. Ginsberg, and W. B. Wood. 1969. Microbiology. Harper and Row, New York.

Ebert, D. 1994. Virulence and local adaptation of a horizontally transmitted parasite. Science 265:1084-1086. - 1998. Experimental evolution of parasites. Science 282: $1432-1435$.

Ebert, D., and W. Hamilton. 1996. Sex against virulence: the coevolution of parasitic diseases. Trends Ecol. Evol. 11:79-82.

Ebert, D., and E. A. Herre. 1996. The evolution of parasitic diseases. Parasitol. Today 12:96-101.

Ebert, D., and K. Mangin. 1997. The influence of host demography on the evolution of virulence of a microsporidian gut parasite. Evolution 51:1828-1837.

Ewald, P. W. 1993. The evolution of virulence. Sci. Am. 268:56-62. Fenner, F., and F. Ratcliffe. 1965. Myxomatosis. Cambridge Univ. press, Cambridge, U.K.

Frank, S. A. 1992. A kin selection model for the evolution of virulence. Proc. R. Soc. Lond. B. 250:195-197.

- 1994 . Recognition and polymorphism in host-parasite genetics. Philos. Trans. R. Soc. B. 346:283-293.

- 1996. Models of parasite virulence. Q. Rev. Biol. 71:37-78.

Gandon, S., and Y. Michalakis. 2000. Evolution of parasite virulence against qualitative or quantitative host resistance. Proc. R. Soc. Lond. B. 267:985-990.

Gandon, S., V. A. Jansen, and M. van Baalen. 2001. Host life history and the evolution of parasite virulence. Evolution 55:1056-1062.

Gupta, S., and A. Hill. 1995. Dynamic interactions in malaria: host heterogeneity meets parasite polymorphism. Proc. R. Soc. Lond. B. 261:271-277.

Levin, B. R., and J. J. Bull. 1994. Short-sighted evoluion and the virulence of pathogenic microorganisms. Trends Microbiol. 2: $76-81$.

Levin, S., and D. Pimentel. 1981. Selection of intermediate rates of increase in parasite-host systems. Am. Nat. 117:308-315.

Lipsitch, M., E. A. Here, and M. A. Nowak. 1995a. Host population structure and the evolution of virulence: a "law of diminishing returns." Evolution 49:747-748.

Lipsitch, M., M. A. Nowak, D. Ebert, and R. M. May. 1995b. The population dynamics of vertically and horizontally transmitted parasites. Proc. R. Soc. Lond. B. 260:321-327.

Mackinnon, M. J., and A. F. Read. 1999. Genetic relationship between parasite virulence and transmission in the rodent malaria Plasmodium chabaudi. Evolution 53:689-703.

May, R. M., and R. M. Anderson. 1983. Epidemiology and genetics in the coevolution of parasites and hosts. Proc. R. Soc. Lond. B. 219:281-313.

May, R. M., and M. A. Nowak. 1995. Coinfection and the evolution of parasite virulence. Proc. R. Soc. Lond. B. 261:209-215.

Messenger, S. L., I. J. Molineux, and J. J. Bull. 1999. Virulence evolution in a virus obeys a trade-off. Proc. R. Soc. Lond. B. 266:397-404

Morand, S., S. D. Manning, and M. E. Woolhouse. 1996. Parasitehost coevolution and geographic patterns of parasite infectivity and host susceptibility. Proc. R. Soc. Lond. B. 263:119-128.

Mosquera, J., and F. R. Adler. 1998. Evolution of virulence: a unified framework for coinfection and superinfection. J. Theor. Biol. 195:293-313. 
Nowak, M. A., and R. M. May. 1994. Superinfection and the evolution of parasite virulence. Proc. R. Soc. Lond. B. 255:81-89.

Regoes, R. R., M. A. Nowak, and S. Bonhoeffer. 2000. Evolution of virulence in a heterogeneous host population. Evolution 54: 64-71.

Schulman, J. L. 1970. Effects of immunity on transmission of influenza: experimental studies. Prog. Med. Virol. 12:128-160.

Traub, E. 1936. The epidemiology of lymphocitic choriomeningitis in white mice. J. Exp. Med. 64:183-200.

van Baalen, M. 1998. Coevolution of recovery ability and virulence. Proc. R. Soc. Lond. B. 265:317-325.

van Baalen, M., and M. Sabelis. 1995. The dynamics of multipe infection and the evolution of virulence. Am. Nat. 146:881-910.

Woolhouse, M. E., C. Dye, J. F. Etard, T. Smith, J. D. Charlwood, G. P. Garnett, P. Hagan, J. L. Hii, P. D. Ndhlovu, R. J. Quinnell, C. H. Watts, S. K. Chandiwana, and R. M. Anderson. 1997. Heterogeneities in the transmission of infectious agents: implications for the design of control programs. Proc. Natl. Acad. Sci. USA 94:338-342.

Zhong, S., and C. Dobson. 1996. Heligmosomoides polygyrus: resistance in inbred, outbred, and selected mice. Exp. Parasitol. $82: 122-131$

Corresponding Editor: H. Ochman

\section{APPENDIX}

The Optimal Growth Rate of the Parasite and the Total Transmission of the Parasite from an Infected Host

Calculating $(\mathrm{dP} / \mathrm{dt}) /(\mathrm{dX} / \mathrm{dt})$ from equation (2) and integrating, we obtain:

$$
\log \left(\frac{P+k}{P_{0}+k}\right)=\frac{r}{s} \log \left(\frac{X}{X_{0}}\right)-\frac{h}{s}\left(X-X_{0}\right)
$$

Noting that the maximal transmission occurs when $P(t)$ approaches the lethal density, $D$, we set $P^{\prime}(t)=0$ and obtain $X(t)=$ $r / h$. Because the maximal transmission occurs at $r=r^{*}$, we find equation (4) with the use of equation (A1) at the limits $P_{0} \ll k, D$ $\gg k$, and $h X_{0} / s \ll 1$. When the total transmission of the parasite from an infected host is proportional to the total density of the parasite, we obtain:

$$
\begin{aligned}
l(r) & =c \int_{0}^{\infty} P(t) d t=c \int_{X_{0}}^{X_{\max }} \frac{P(t)+k}{s X} d X \\
& =\frac{c\left(P_{0}+k\right)}{h X_{0}} e^{h X_{0} / s}\left(\frac{s}{h X_{0}}\right)^{r / s-1} \int_{h X_{0} / s}^{h X_{\max } / s} y^{r / s-1} e^{-y} d y .
\end{aligned}
$$

At the approximations $P_{0} \ll k$ and $h X_{0} / s \ll 1$, equation (A2) can be simplified:

$$
l(r)=\frac{k c}{s}\left(\frac{s}{h X_{0}}\right)^{r / s} \int_{0}^{h X_{\max } / s} y^{r / s-1} e^{-y} d y .
$$

When $r<r^{*}$ ( $r^{*}$ defined by eq. 4), an infected host clears the infection by a greatly expanding population of immune cells; therefore, at the approximation $h X_{\max } / s \gg 1$, we obtain:

$$
l(r) \approx \frac{k c}{s}\left(\frac{s}{h X_{0}}\right)^{r / s} \int_{0}^{\infty} y^{r / s-1} e^{-y} d y=\frac{k c}{s}\left(\frac{s}{h X_{0}}\right)^{r / s} \Gamma\left(\frac{r}{s}\right),
$$

where $\Gamma(x)$ is the Euler gamma function. Note that $\Gamma(x)=(x-1)$ ! for integral $x$. When $r \gg r^{*}$, we can assume that immune cells do not expand; approximating $\exp (-y)$ at $y \in\left(0, h X_{\max } / s \ll 1\right)$ as one, we obtain the following expression for the total transmission of the parasite from an infected host at $r \gg r^{*}$ :

$$
l(r)=c \frac{D}{r}
$$

\section{The Duration of Acute Infection}

We consider the duration of acute infection for the cases when $r<r^{*}$ and $r>r^{*}$ separately ( $r^{*}$ is given by eq. 4 ).

If $r>r^{*}$, the parasite kills the host; approximating that the parasite population grows exponentially until it kills the host, the duration of acute infection, $\Delta(r, D)$, will be:

$$
\Delta(r, D) \approx \frac{\ln \left(D / P_{0}\right)}{r} .
$$

If $r<r^{*}$, then the parasite is cleared by the immune response; the duration of acute infection is found by integrating the equation for $d X / d t$ in equation (2):

$$
\Delta(r, D)=\int_{X_{0}}^{X_{\max }} \frac{P+k}{s X P} d X
$$

where the relationship between the number of immune cells, $X$, and parasite density, $P$, at any given moment is given in the equation (A1). Substituting $P(X)$ from equation (A1), the duration of the acute infection when $r<r^{*}$ equals:

$$
\Delta(r, D)=\int_{X_{0}}^{X_{\max }} \frac{d x}{s x}\left[1-\frac{k}{P_{0}+k}\left(\frac{X_{0}}{x}\right)^{r / s} e^{h\left(x-X_{0}\right) / s}\right]^{-1},
$$

where the maximum density of immune cells $\left(X_{\max }\right)$ is approximately found in the equation: $r \ln \left(X_{\max } / X_{0}\right) \approx h\left(X_{\max }-X_{0}\right)$. Summarizing, the duration of acute infection is:

$$
\Delta(r, D)= \begin{cases}\frac{\ln \left(D / P_{0}\right)}{r}, \quad \text { if } D<k\left(\frac{r}{h e X_{0}}\right)^{r / s}, \\ \int_{X_{0}}^{X_{\max }} \frac{d x}{s x}\left[1-\frac{k}{P_{0}+k}\left(\frac{X_{0}}{x}\right)^{r / s} e^{h\left(x-X_{0}\right) / s}\right]^{-1}, \\ \text { otherwise. }\end{cases}
$$

If $f(D)$ describes the distribution of lethal densities, $D$, in the host population then the average duration of acute infection corresponding this distribution will be:

$$
\Delta(r)=\int_{0}^{\infty} \Delta(r, D) f(D) d D
$$

\section{Virulence Defined by the Case Mortality}

The case mortality is the probability of host death due to the infection. In a host population with heterogeneity defined by the $f(D)$, the average case mortality, $M(r)$, can be calculated as follows. The maximum density reached by a parasite with the growth rate $r$ during acute infection in equation (2) is given by equation (4), that is, $P_{\max } \approx k\left(r /\left(h e X_{0}\right)\right)^{r / s}$. Therefore, hosts with threshold densities less than $P_{\max }\left(D<P_{\max }\right)$ will die when infected with such a parasite strain, whereas others will survive. Thus, the case mortality will be:

$$
m(r, D)= \begin{cases}1, & \text { if } D<k\left(\frac{r}{h e X_{0}}\right)^{r / s} \\ 0, & \text { otherwise. }\end{cases}
$$

The average case mortality rate, $M$, is simply equal to the fraction of the host population with threshold densities less than the maximum density, $P_{\max }$, reached by the parasite:

$$
M(r)=\int_{0}^{\infty} m(r, D) f(D) d D=\int_{0}^{P_{\max }} f(D) d D .
$$


Virulence Defined by the Lethal Density, $L D_{50}$

The $L D_{50}$ is the initial dose of the parasite required to kill $50 \%$ of infected hosts in a fixed period of time (Davis et al. 1969). We derive the $L D_{50}$ when this period of time is greater than (or equal to) the duration of acute infection. The results obtained under this condition are similar to those found when the fixed period is shorter (not shown).

We calculate the $L D_{50}$ as follows. The maximal density obtained by a parasite with growth rate $r$ during the course of acute infection is given in equation (A1):

$$
P_{\max } \approx\left(P_{0}+k\right)\left(\frac{r}{h e X_{0}}\right)^{r / s}
$$

The average case mortality is defined in equation (A12), with
$P_{\max }$ from the equation (A13). Because the initial size of the parasite population is now variable, we define $L D_{50}$ as the density, $P_{0}$, at which the case mortality, $M$, is one-half:

$$
L D_{50}(r)=P_{0}: \quad \int_{0}^{\left(P_{0}+k\right)\left(r /\left(h e X_{0}\right)\right)^{r / s}} f(D) d D=\frac{1}{2} .
$$

Solving this integral equation for a given distribution $f(D)$ provides an estimate for $L D_{50}$. When the variance of the distribution $f(D)$ is small, the solution of equation (A14) is approximately:

$$
L D_{50}(r) \approx\langle D\rangle\left(\frac{h e X_{0}}{r}\right)^{r / s}-k,
$$

where $\langle D\rangle$ is the average lethal density in the host population. 